UUITP-15/02

hep-th/0211006

\title{
Can MAP and Planck map Planck physics?
}

\author{
Lars Bergström, ${ }^{1}$ and Ulf H. Danielsson, ${ }^{2}$ \\ ${ }^{1}$ Department of Physics, Stockholm University, AlbaNova University Center \\ S-106 91 Stockholm, Sweden \\ ${ }^{2}$ Institutionen för Teoretisk Fysik, Box 803, SE-751 08 Uppsala, Sweden \\ lbe@physto.se, ulf@teorfys.uu.se
}

\begin{abstract}
We investigate whether a recently proposed modulation of the power spectrum of primordial density fluctuations generated through transplankian (maybe stringy) effects during inflation can be observed. We briefly review the mechanism leading to the modulation and apply it to a generic slow-roll scenario of inflation. We then investigate how these primordial modulation effects leave an imprint in the cosmic microwave background radiation. Our conclusions are that for favourable parameter values already the presently flying MAP satellite will have a chance to detect such transplanckian oscillations in the pattern of temperature fluctuations on the sky, and that the upcoming Planck satellite will either detect them or put stringent limits related to the mass scale where the new effects appear.
\end{abstract}

October 2002 


\section{Introduction}

It is an intriguing possibility that effects of physics beyond the Planck scale might be visible on cosmological scales in the spectrum of the cosmic microwave background (CMBR) fluctuations. The physical mechanism that could make this possible is inflation. Inflation magnifies microscopic quantum fluctuations into cosmic size, and thereby provides seeds for structure formation. The details of physics at the highest energy scales is therefore reflected in the distribution of galaxies and other structures on large scales.

In the standard inflationary scenario, initial conditions for the inflaton field are imposed in the infinite past when the modes are infinitely small. In this limit the effect of the inflationary horizon and the expansion of the universe can be ignored, the space time is essentially Minkowski, and there is a unique vacuum for the inflaton field. We will refer to this vacuum as the Bunch-Davies vacuum. The problem with this reasoning is that we are assuming that intuition based on field theory is always applicable and that nothing dramatic happens at the smallest length scales, when stringy or transplankian physics is likely to occur. Clearly this is an assumption that needs to be carefully scrutinized. Could there be effects of new physics that will change the predictions of inflation? In particular one could worry about changes in the predictions of the CMBR fluctuations. Several groups have investigated various ways of modifying high energy physics in order to look for such modifications, [1-33].

Without a good understanding of physics near the Planck, or string, scale, one can also take a purely empiricist point of view, as advocated in [27, and simply encode the new physics in the choice of initial conditions when a mode of the inflaton has a wavelength comparable to the scale where new physics is expected. (See also [11.) Since the mode will not be infinitely small compared to the inflationary scale, the time dependence of the background will be important and the choice of initial conditions not unique. The effects on physics at lower energies will then be encoded in the choice of a vacuum not necessarily the same as the Bunch-Davies vacuum.

As will be reviewed in Section 2 the general expectation is that the effects will be of linear order in $H / \Lambda$, where $H$ is the Hubble constant during inflation and $\Lambda$ the scale where new physics appears. Smaller corrections would suggest some kind of fine tuning and require knowledge of planckian physics in order to be justified. The Bunch-Davies vacuum is still a vacuum candidate but, contrary to the case where the initial conditions are imposed in the infinite past, it is not the unique choice that one can argue for.

There has been extensive discussions of these results in the literature. It has been known since a long time that there is a family of vacua in de Sitter space that respects all the symmetries, the $\alpha$-vacua, 34] 355 37 [38] 39. As pointed out in [29] the initial condition approach to the transplanckian problem allows for a discussion of many of the transplanckian effects in terms of these vacua. In fact, in [40] 41] [42] it has been pointed out that there could be tricky problems with field theories based on non trivial vacua of this sort. In particular loop amplitudes are not necessarily 
well defined. But as argued in [32] (see also [33] for a similar point if view), the real world is not expected to correspond to field theoretic $\alpha$-vacua up to infinite energies. The best way to understand this is simply to view the system as an excitation above the Bunch-Davies vacuum. What happens above the fundamental scale we can only speculate about, but the hope is that the size, and as we will see even the qualitative form, of the corrections (if there are any) can be obtained without detailed knowledge of transplanckian physics.

The fact the effects are linear in $H / \Lambda$ is of crucial importance. If the effects would come in at a higher power they will not be detectable unless we invoke more exotic models of high energy physics involving large extra dimensions. Discussions of such possibilities can be found in [24]. What we will argue for here, is that rather conservative models based on naturally occurring scales and expectations from string theory give rise to effects that could be within reach.

At this point one should note, however, that even if we focus on linear effects, there are nevertheless at least two different ways in which they might be detectable. Either through corrections to the CMBR fluctuations, or through high energy gamma rays. As discussed in [43], if one assumes that similar vacuum selection effects take place today as during inflation - after all the universe is still expanding - this would lead to the production of high energy radiation in the present universe. It is argued in 43 that this already puts stringent limits on possible effects on the CMBR. While there is a certain tension between these results we believe that the extrapolation over many orders of magnitude from the inflationary period to the present day universe is sufficiently uncertain that one can not make definite statements at the present time. The most reasonable strategy seems to be to pursue both approaches in looking for detectable effects.

To summarize, we believe that a detailed analysis of the possible effects on the CMBR is of great interest in view of the upcoming MAP and Planck satellites, and this is also the subject of the present paper.

The organization of the paper is as follows. In Section 2 we review the choice of initial conditions that we propose to investigate. In Section 3 we make some rough estimates of the kind of signatures that one can expect from reasonable high energy physics. In Section 1 we numerically analyze the corrections to the CMBR spectrum and discuss whether the effects can be detected by MAP or Planck. We end, finally, with some conclusions.

\section{Review of setup}

In this section, following [27], we will give a typical example of the kind of corrections one might expect due to changes in the low energy quantum state of the inflaton field due to transplanckian effects. First we need to find out when to impose the initial conditions for a mode with a given (constant) comoving momentum $k$. To do this it is convenient to use conformal coordinates rather than the standard Robertson-Walker 
coordinates where the inflating metric is given by

$$
d s^{2}=d t^{2}-a(t)^{2} d x^{2}
$$

with the scale factor given by $a(t)=e^{H t}$. The conformal coordinates are obtained by defining $\eta=-\frac{1}{a H}$ and the metric takes the form

$$
d s^{2}=\frac{1}{H^{2} \eta^{2}}\left(d \eta^{2}-d \mathbf{x}^{2}\right)
$$

We now note that the physical momentum $p$ and the comoving momentum $k$ are related through

$$
k=a p=-\frac{p}{\eta H}
$$

and impose the initial conditions when $p=\Lambda$, where $\Lambda$ is the energy scale important for the new physics. This scale could be the Planck scale or the string scale. We find that the conformal time when the initial condition is imposed to be

$$
\eta_{0}=-\frac{\Lambda}{H k}
$$

As we see, different modes will be created at different times, with a smaller linear size of the mode (larger $k$ ) implying a later time.

To proceed we need the equation of motion for the scalar field (ignoring the potential)

$$
\ddot{\phi}+3 H \dot{\phi}-\nabla^{2} \phi=0
$$

which in terms of the conformal time $\eta$, and the rescaled field $\mu=a \phi$, becomes

$$
\mu_{k}^{\prime \prime}+\left(k^{2}-\frac{a^{\prime \prime}}{a}\right) \mu_{k}=0 .
$$

A nice discussion of the quantization of the system can be found in [44. In terms of time dependent oscillators we can write

$$
\begin{aligned}
& \mu_{k}(\eta)=\frac{1}{\sqrt{2 k}}\left(a_{k}(\eta)+a_{-k}^{\dagger}(\eta)\right) \\
& \pi_{k}(\eta)=\mu_{k}^{\prime}(\eta)+\frac{1}{\eta} \mu_{k}(\eta)=-i \sqrt{\frac{k}{2}}\left(a_{k}(\eta)-a_{-k}^{\dagger}(\eta)\right)
\end{aligned}
$$

which also can be expressed in terms of oscillators at a specific moment using the Bogolubov transformation

$$
\begin{aligned}
a_{k}(\eta) & =u_{k}(\eta) a_{k}\left(\eta_{0}\right)+v_{k}(\eta) a_{-k}^{\dagger}\left(\eta_{0}\right) \\
a_{-k}^{\dagger}(\eta) & =u_{k}^{*}(\eta) a_{-k}^{\dagger}\left(\eta_{0}\right)+v_{k}^{*}(\eta) a_{k}\left(\eta_{0}\right)
\end{aligned}
$$


We find

$$
\mu_{k}(\eta)=f_{k}(\eta) a_{k}\left(\eta_{0}\right)+f_{k}^{*}(\eta) a_{-k}^{\dagger}\left(\eta_{0}\right)
$$

where

$$
f_{k}(\eta)=\frac{1}{\sqrt{2 k}}\left(u_{k}(\eta)+v_{k}^{*}(\eta)\right)
$$

is a solution of the mode equation (6). The solution can be written as the linear combination

$$
f_{k}=\frac{A_{k}}{\sqrt{2 k}} e^{-i k \eta}\left(1-\frac{i}{k \eta}\right)+\frac{B_{k}}{\sqrt{2 k}} e^{i k \eta}\left(1+\frac{i}{k \eta}\right)
$$

where

$$
\left|A_{k}\right|^{2}-\left|B_{k}\right|^{2}=1
$$

We are now in the position to start discussing the choice of vacuum. Without knowledge of the high energy physics we can only list various possibilities and investigate whether there is a typical size or signature of the new effects. As an example, we will focus on a choice of vacuum determined by

$$
a_{k}\left(\eta_{0}\right)\left|0, \eta_{0}\right\rangle=0
$$

This vacuum should be viewed as a typical representative of other vacua besides the Bunch-Davies. It can be characterized as a vacuum corresponding to a minimum uncertainty in the product of the field and its conjugate momentum, 44, the vacuum with lowest energy (lower than the Bunch-Davies) [11], or as the instantaneous Minkowski vacuum. Therefore, it is as special as the Bunch Davies vacuum and there is no a priori reason for planckian physics to prefer one over the other.

We note that we in general have a class of different vacua depending on the parameter $\eta_{0}$. At this initial time it follows from Eq. (8) that $v_{k}\left(\eta_{0}\right)=0$, which, with the help of Eq. (7) and Eq. (11), will constrain $A_{k}$ and $B_{k}$ to obey

$$
B_{k}=\frac{i e^{-2 i k \eta_{0}}}{2 k \eta_{0}+i} A_{k}
$$

Note that for $\eta_{0} \rightarrow-\infty$ one finds $A_{k}=1$ and $B_{k}=0$ which means that the BunchDavies vacuum is recovered. The reason that the new vacuum is de Sitter invariant has to do with the way that the initial conditions for a mode are imposed. The crucial point is that this is done at a fixed scale, not a fixed time, and as a consequence physics will actually be independent of time (up to changes in the inflationary cosmological constant).

We are now in a position to calculate the expected fluctuation power spectrum:

\footnotetext{
${ }^{1}$ In [30] it is argued, based on adiabaticity, that even if linear corrections are fine tuned away there will nevertheless remain corrections of order $(H / \Lambda)^{3}$ compared to the Bunch Davies.
} 
$P(k)=\left(\frac{H}{\dot{\phi}}\right)^{2}\left\langle\left|\phi_{k}(\eta)\right|^{2}\right\rangle=\left(\frac{H}{\dot{\phi}}\right)^{2} \frac{1}{a^{2}}\left\langle\left|\mu_{k}(\eta)\right|^{2}\right\rangle=\left(\frac{H}{\dot{\phi}}\right)^{2}\left(\frac{H}{2 \pi}\right)^{2}\left(1-\frac{H}{\Lambda} \sin \left(\frac{2 \Lambda}{H}\right)\right)$.

This result should be viewed as a typical example of what to be expected from transplanckian physics if we allow for effects which at low energies reduce to changes compared to the Bunch-Davies case. We note that the size of the correction is linear in $H / \Lambda$, and that a Hubble constant that varies during inflation give rise to a modulation of the spectrum. As argued in [27], this is presumably a quite generic effect that is present regardless of the details of the transplanckian physics. (See also [30] for a discussion about this). After being created at the fundamental scale the modes oscillate a number of times before they freeze. The number of oscillations depend on the size of the inflationary horizon and therefore changes when $H$ changes.

\section{What to look for}

As discussed in the previous section, a Hubble constant that does not vary during inflation would just imply a small change in the overall amplitude of the fluctuation spectrum which would not constitute a useful signal. Luckily, since the Hubble constant is expected to vary, the situation is much more interesting. [?

We will discuss what happens using slow roll parameters, see, e.g., 445, where in particular,

$$
\varepsilon=\frac{M_{p l}^{2}}{2}\left(\frac{V^{\prime}}{V}\right)^{2}
$$

with $M_{p l}=1 / \sqrt{8 \pi G} \sim 2 \cdot 10^{18} \mathrm{GeV}$ as the (reduced) Planck mass. It is not difficult to show (using that $H$ is to be evaluated when a given mode crosses the horizon, $k=a H)$ that

$$
\frac{d H}{d k}=-\frac{\varepsilon H}{k}
$$

which gives

$$
H \sim k^{-\varepsilon}
$$

The $k$ dependence of $H$ will translate into a modulation of $P(k)$, with a periodicity given by

$$
\frac{\Delta k}{k} \sim \frac{\pi H}{\varepsilon \Lambda}
$$

The overall amplitude of the CMBR spectrum (at the largest scales, still outside the

\footnotetext{
${ }^{2}$ In [28] the case of slow roll was also studied. For our purposes, however, a small $\varepsilon$ expansion around the de Sitter case is adequate.
} 
causal horizon at the time of photon decoupling) is given by

$$
\left(\frac{H}{\dot{\phi}}\right)^{2}\left(\frac{H}{2 \pi}\right)^{2}=\frac{1}{24 \pi^{2} M_{p l}^{4}} \frac{V}{\varepsilon},
$$

which is restricted through measurements according to

$$
\frac{V^{1 / 4}}{\varepsilon^{1 / 4}} \sim 0.027 M_{p l} \equiv \beta M_{p l}
$$

Using the Friedmann equation one finds

$$
H^{2}=\frac{V}{3 M_{p l}^{2}} \sim \frac{\beta^{4}}{3} M_{p l}^{2} \varepsilon,
$$

from which it follows that

$$
\frac{H}{M_{p l}} \sim \frac{\beta^{2} \varepsilon^{1 / 2}}{\sqrt{3}} \sim 4 \cdot 10^{-4} \sqrt{\varepsilon} .
$$

We now put the scale where initial conditions are imposed to be

$$
\Lambda=\gamma M_{p l}
$$

which implies that

$$
\frac{\Delta k}{k} \sim \frac{\pi H}{\varepsilon \gamma M_{p l}} \sim \frac{\pi \beta^{2}}{\sqrt{3} \gamma \sqrt{\varepsilon}} \sim 1.3 \cdot 10^{-3} \frac{1}{\gamma \sqrt{\varepsilon}}
$$

and

$$
\xi \equiv \frac{H}{\Lambda} \sim 4 \cdot 10^{-4} \frac{\sqrt{\varepsilon}}{\gamma} .
$$

To be more specific, we will now consider a realistic example. In the Horava-Witten model [46] [47], unification occurs roughly at the same scale as a fifth dimension becomes visible and also comparable to the string scale and the higher dimensional Planck scale. For a discussion and references see, e.g., [48] or [24]. As a rough estimate we therefore put $\Lambda=2 \cdot 10^{16} \mathrm{GeV}$. This is a rather reasonable possibility within the framework of the heterotic string and corresponds to $\gamma=0.01$. The Hubble constant during inflation can not be much larger than $H=7 \cdot 10^{13} \mathrm{GeV}$, corresponding to $\varepsilon=0.01$. Using these values we find

$$
\begin{aligned}
\xi & \sim 0.004 \\
\frac{\Delta k}{k} & =\Delta \ln k \sim 1 .
\end{aligned}
$$

This means one oscillation per logarithmic interval in $k$, which should be visible in high-precision CMBR observation experiments. In the next section we will consider these predictions in more detail. 


\section{Predictions for CMBR measurements}

Density perturbations generated during the inflationary phase will imprint corresponding temperature fluctuations in the cosmic microwave background radiation (CMBR). These temperature fluctuations on the sky which are gaussian due to their origin in oscillations of the inflation field can be characterized by their angular power spectrum. (For a review, see e.g. [49]). Expanding the temperature fluctuation in spherical harmonics (excluding the dipole, which is indistinguishable from effects caused by our motion with respect to the cosmic rest frame),

$$
\frac{\Delta T}{T}(\theta, \phi)=\sum_{\ell=2}^{\infty} \sum_{m=-\ell}^{m=\ell} a_{\ell m} Y_{\ell m}(\theta, \phi)
$$

the angular power spectrum $C_{\ell}$ is defined by

$$
\left\langle a_{\ell m}^{*} a_{\ell^{\prime} m^{\prime}}\right\rangle=C_{\ell} \delta_{\ell \ell^{\prime}} \delta_{m m^{\prime}}
$$

where the Kronecker deltas appear thanks to statistical isotropy. Since we only have one sky to observe, an unbiased estimator for the angular power spectrum, assuming full sky coverage, is given by

$$
C_{\ell}=\frac{1}{2 \ell+1} \sum_{m=-\ell}^{m=\ell} a_{\ell m}^{*} a_{\ell m} .
$$

Well-known physics in the radiation dominated era and at the epoch near photon decoupling will modify the "primordial" power spectrum in momentum space $P(k)$ through a transfer function to the measured angular power spectrum $C_{\ell}$. In particular, there will be a series of "acoustic peaks", of which the location of the first one is an excellent indicator of the overall geometry of the Universe. In the past few years, a number of balloon-borne and terrestrial experiments have mapped out an ever larger region in $\ell$-space. In particular, the location of the first peak has been determined by the experiments BOOMERANG [50], MAXIMA [51] and DASI [52 to give clear evidence for a nearly flat Universe. This has very recently been confirmed with even higher accuracy by Archeops [53, and consistency of the CMBR data with the standard scenario, including the inflationary mechanism, has been further strengthened by the recent detection of a polarization signal by DASI [54]. The satellite experiment MAP [55] is expected to give results soon, and a very ambitious mission, the Planck satellite, is being built for a scheduled launch in 2007 [56].

It is expected that the measurements of the angular power spectrum of the CMBR encoded in the measured set of $C_{\ell}$ from satellites like Planck, although superior to all present measurements, at most will reach an accuracy of $10^{-2}$ for a particular value of $\ell$. This is essentially set by cosmic variance, which is the fact that we only can sample one sky from one particular point of observation (see Eq. 29). This makes it impossible

[57] to reach a relative accuracy better than about $1 / \sqrt{(2 \ell+1) f_{\text {sky }}}$, where $f_{\text {sky }}$ is the 
fraction of the sky covered by the experiment (which, for satellite experiments, will be close to unity - around 0.8 for Planck). This stochastic limitation of the attainable accuracy is an order of magnitude above the estimate of the transplanckian signal of the previous section. However, one has to take into account the fact that future CMBR satellites will measure thousands of independent data points corresponding to different $\ell$ values.

The transplanckian effects, regardless of their precise nature, have a rather generic signature in form of their modulation of the spectrum. This means that these effects will not likely be degenerate with a change in the power spectrum caused by uncertainties in other parameters such as the slope of the primordial spectrum $P(k)$ (which gives a nearly monotonous change with $\ell$ of the angular power spectrum $C_{\ell}$ ) or the baryon fraction of the matter density (which also causes oscillatory behavior in the angular power spectrum, but with the modulation closely tied to the location of the acoustic peaks). If it had just been an overall shift or tilt of the amplitude it would not have been possible to measure the effect even if it had been considerably larger than the percentage level. The shift would just have gone into a changed value or slope of, e.g., $H$. With a definite signature we can use several measurement points throughout the spectrum, as we now discuss. We will see that Planck might be able to detect transplanckian effects at the $10^{-3}$ level, which would put the Horava-Witten model within range, or at least tantalizingly close. (To make a more definitive statement, a more careful analysis of covariance of the transplanckian signature with a large number of other cosmological parameters will be needed, something we leave for future work.)

Let us now proceed with a somewhat more careful analysis. We see from Eq. (26) that, for fixed $\varepsilon$, it is reasonable to expect a range of values of $\gamma$ within the reach of Planck (or even MAP). Too large $\gamma$ and the amplitude will be too small. Too small $\gamma$ and the period will be too long, but in an intermediate region the effects should be observable.

In our result for the transplanckian power spectrum, Eq. (15)

$$
P(k)=\left(\frac{H}{\dot{\phi}}\right)^{2}\left(\frac{H}{2 \pi}\right)^{2}\left(1-\frac{H}{\Lambda} \sin \left(\frac{2 \Lambda}{H}\right)\right),
$$

we can for simplicity assume that the product of the first and second factors gives a scale-invariant spectrum, i.e.,

$$
\left(\frac{H}{\dot{\phi}}\right)^{2}\left(\frac{H}{2 \pi}\right)^{2}=\text { const. }
$$

(This assumption is not crucial, but can be arranged by a suitable choice of the inflaton potential parameters defining the slow-roll phase of inflation.) We can then instead of the parameter pair $\varepsilon$ and $\gamma$ choose the pair $\varepsilon$ and $\xi=\frac{H_{n}}{\Lambda}$, with $H_{n}$ the Hubble parameter evaluated when some particular scale $k_{n}$ leaves the horizon (in practice, 
we will choose this scale to correspond to the largest angular scales measurable in the CMBR). This gives

$$
\frac{H}{\Lambda}=\xi\left(\frac{k}{k_{n}}\right)^{-\varepsilon},
$$

with $\xi \sim 4 \cdot 10^{-4} \frac{\sqrt{\varepsilon}}{\gamma}$, which enables us to parametrize the power spectrum in terms of the small parameter $\xi$ as

$$
P(\varepsilon, \xi ; k)=P_{0}(k)\left(1-\xi\left(\frac{k}{k_{n}}\right)^{-\varepsilon} \sin \left[\frac{2}{\xi}\left(\frac{k}{k_{n}}\right)^{\varepsilon}\right]\right) .
$$

Here $P_{0}(k)$ is a scale-invariant spectrum which we can use for comparison in the numerical work. The advantage of using $\xi$ instead of $\gamma$ is that it is a small parameter ( $\xi \sim 0.004$ in the Horrava-Witten case) which can be safely extrapolated to zero - the transplanckian effects will have an unobservably small amplitude in this limit. The possible variation of $\varepsilon$ is limited by the overall normalization of the observed temperature fluctuations, so effectively one can choose to regard the transplanckian effects as being a one-parameter family of modulating functions, with amplitude determined by the value of $\xi$. Since the parameter $\gamma$ has a simpler physical interpretation, we will however also use that one in the discussion of our results.

In Fig. 1 (a) and (b) we show examples of power spectra $P(k)$ for various values of $\gamma$, (and therefore of $\xi$ ) for $\varepsilon=0.01$ and 0.03 , respectively.
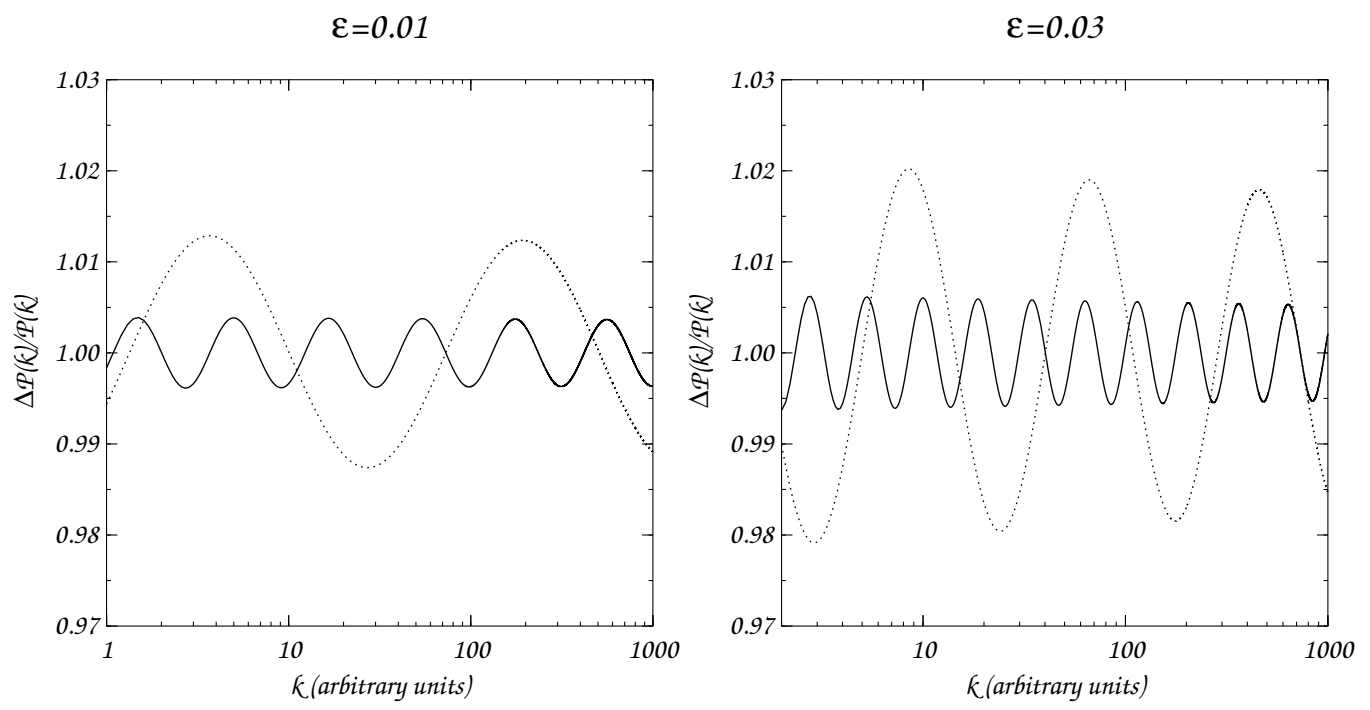

Figure 1: (a) The modulation of the power spectrum of primordial density fluctuations predicted in the transplanckian model (Eq. (15)) with the parameter $\varepsilon=0.01$ and $\gamma=0.01$ (solid line) and $\gamma=0.003$ (dotted line). (b) Same as in (a) but with $\varepsilon=0.03$.

To connect to observable quantities we have to compute the angular power spectrum $C_{\ell}$ for each set of parameters. This is a complicated task which has to be per- 
formed numerically. Fortunately, the form of the transplanckian modulation makes it easy to modify existing computer codes to incorporate the effects. We will use the well-known cmbfast code, which is publicly available 58. As a first illustration of the effects one may expect in the angular power spectrum of the CMBR, we show in Fig. 2 how the transplanckian effects using the Hořava-Witten parameters $(\varepsilon=0.01, \gamma=0.01$ meaning $\xi=0.004)$ will appear, as well as a slightly lower $\gamma$, 0.003, $(\xi=0.013)$ which gives a larger effect. Shown is the fractional change $\Delta C_{\ell} / C_{\ell}$ as a function of $\ell$, where we always will compare with an "unperturbed" model with scale-invariant power spectrum, present Hubble constant $65 \mathrm{~km} / \mathrm{s} \mathrm{Mpc}^{-1}$, matter density parameter $\Omega_{M}=0.3$ of which baryons contribute 0.045 and Cold Dark Matter 0.255 , present cosmological constant contribution $\Omega_{\Lambda}=0.7$, and no reionization. Since we only will investigate small changes around a given model, the exact choice of parameters for the reference model is not essential. Also, for the same reason we consider it safe to display the trends caused by the transplanckian modulation although they often are below the nominal accuracy of the cmbfast program, which is around a percent or so. (More accurate codes will no doubt appear well in time before the launch of Planck.) The scale for $\ell$ in Fig. 2 (a) is logarithmic to show that much of the modulation apparent in Fig. 11 has survived being submitted by the transfer function to the CMBR angular power spectrum.

For comparison, we also show in the same Figure the changes caused by a tilt of the power spectrum by 0.001, and a change in the baryon density (or $\Omega_{b} h^{2}$, which is a more relevant physical quantity) by one percent (keeping the total matter density unchanged). As can be seen, the effects caused by the transplanckian modulation are visibly very different from the other ones (we have verified that this is the case also for the other "standard" CMBR parameters). In Fig. \& (b) the same results are shown using instead a linear scale for $\ell$.

We now want to get a quantitative measure of the potential observability of the effects. The machinery of extracting cosmological parameters from the CMBR is well developed by now (see, e.g., [59, 60, 61, 62, 63, 64, 65]). Given a "true" underlying cosmology with a definite set of parameter values $s_{i}^{0}, i=1, \ldots N$, the probability to measure a given set of $C_{\ell}$ corresponding to fitted parameters $s_{i}$, which deviate from the true values by $\delta s_{i}=s_{i}-s_{i}^{0}$, can be computed from the likelihood function

$$
\mathcal{L}=\mathcal{L}_{m} e^{-\frac{1}{2} \sum_{i j} F_{i j} \delta s_{i} \delta s_{j}},
$$

where the "Fisher matrix" is given by

$$
F_{i j}=\sum_{\ell} \frac{1}{\left(\Delta C_{\ell}^{\text {obs }}\right)^{2}} \frac{\partial C_{\ell}}{\partial s_{i}} \frac{\partial C_{\ell}}{\partial s_{j}} .
$$

Here the variance $\left(\Delta C_{\ell}^{\text {obs }}\right)^{2}$ gets a contribution both from the cosmic variance discussed above and from the instrumental noise related to the angular resolution and sensitivity of the experiment: 

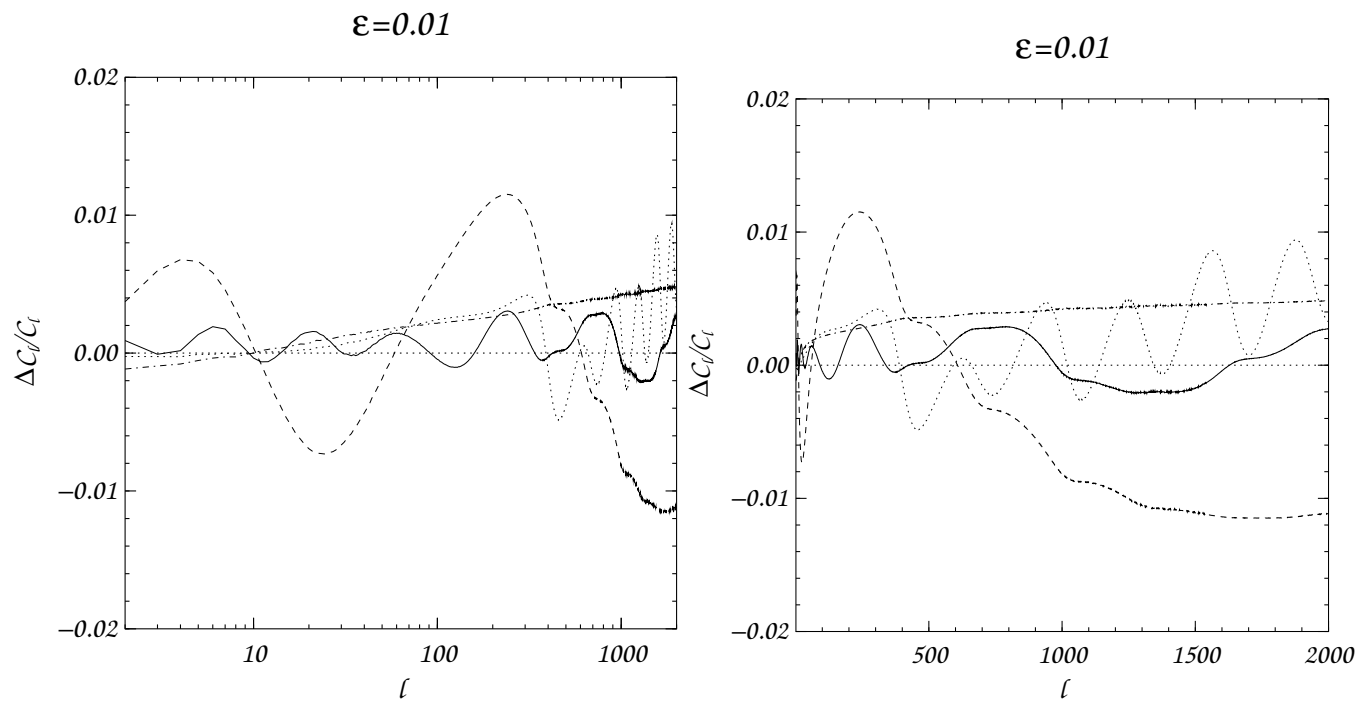

Figure 2: (a) The relative change of the CMBR angular power spectrum $\Delta C_{\ell} / C_{\ell}$ caused by transplanckian modulation of the primordial power spectrum with $\varepsilon=$ 0.01 and $\gamma=0.01$ (solid line) and $\gamma=0.003$ (dashed line). For comparison, the corresponding change caused by a change of the scale invariant spectrum $n=1$ to a slightly bluer one with $n=1.001$ is shown (the almost straight, monotonous dashdotted line). Also shown is the effect of increasing the baryon fraction $\Omega_{b}$ by one percent, from 0.045 to 0.04545 (dotted line). The scale in $\ell$ is logarithmic to facilitate comparison with the modulation of the primordial spectrum shown in Fig. [1. The small irregularities of the curves is caused by numerical noise in the computations. (b) Same as (a), but with linear scale in $\ell$.

$$
\left(\Delta C_{\ell}^{\mathrm{obs}}\right)^{2} \sim \frac{2}{(2 \ell+1) f_{\mathrm{sky}}}\left(C_{\ell}+D_{\ell}\right)^{2},
$$

where

$$
D_{\ell}=\frac{1}{\sum_{c} w_{c} e^{-\ell(\ell+1) / \ell_{c}^{2}}} .
$$

The experimental quantities $w_{c}$ and $\ell_{c}$ depend on the frequency channel $c$ of the instrument. For Planck, we will use the first three HFI channels with parameters as given in [60].

As a first reality check of the observability of the transplanckian modulation we assume that all other parameters are kept at the default values, and we use hypothetical measurements from the Planck satellite to determine with which significance we can say for a given value of $\varepsilon$ (which is more or less determined by the overall magnitude of the fluctuations on the largest, COBE, scales) that $\xi$ is different from 
zero. In this case the likelihood function collapses to

$$
\mathcal{L} / \mathcal{L}_{m}=e^{-\frac{1}{2} \sum_{\ell}\left(\frac{\Delta C_{\ell}^{\xi}}{\Delta C_{\ell}^{\text {obs }}}\right)^{2}},
$$

where $\Delta C_{\ell}^{\xi}$ is the deviation from the reference model caused by the modulation. For the Hořava-Witten values of the parameters $(\varepsilon=0.01, \xi=0.004)$ we find that in this (idealized) case of knowing all other parameters, the effect is right at the limit of being observable with the likelihood of a chance occurrence of such a modulation being around $17 \%$. For a slightly larger value of $\xi$, which can be obtained by a larger $\varepsilon$ and/or smaller $\gamma$, the transplanckian modulation would be clearly visible. For instance, with the same value of $\varepsilon$ and $\gamma=0.003$, the effect would have a significance of around 10 standard deviations. Likewise, for $\varepsilon=0.03$ and $\gamma=0.01$, the significance is around 3 standard deviations. For the favorable case $\varepsilon=0.03$, $\gamma=0.003$ (or $\xi=0.02$, still well within reasonable bounds of what one can expect for the unknown transplanckian physics) the significance of the modulation would approach 16 standard deviations.

These estimates makes us believe that transplanckian effects, for $\xi$ values not too much smaller than 0.01 , will turn out to be observable also after a more complete analysis which includes a full Fisher matrix treatment of covariance of parameters. In fact, by inspection of Fig. \& we see that the effects for $\varepsilon=0.01$ and $\gamma=0.003$ $(\xi \sim 0.013)$ are visibly larger than those obtained by changing the baryon fraction by $1 \%$, and the latter is definitely within reach of the Planck satellite as shown in the analysis of [60].

To make a first step towards a more complete analysis, we have computed the 2 by 2 Fisher matrix obtained by letting both $\xi$ and the only other quantity which causes a modulation - although of a very different type as we have seen in Fig 2 namely $\Omega_{b}$ vary. We confirm that also in this case the one-sigma exclusion limit for $\xi$ is of the order of 0.002 .

For the case $\varepsilon=0.03, \gamma=0.003(\xi=0.023)$, the effects could already start to be noticeable in the MAP experiment. Using the parameters listed as $\mathrm{MAP}^{+}$in [60], we obtain a calculated significance between 3 and 4 standard deviations. To judge whether this is observable in the real MAP experiment, a more realistic treatment of both the experimental sensitivity and the effects of other cosmological parameters would be called for. Doing the simple 2 by 2 Fisher matrix analysis again, we find that the effect still is visible, but with only around 2 sigma confidence. In any case, we find it intriguing that transplanckian effects may be at the doorstep of current CMBR observations.

Thus, the CMBR may hold the clue to one of the most exciting problems of today's theoretical physics, that of what happens to space-time for distances smaller than the string or Planck lengths. 


\section{Conclusions}

We have shown that in one of the simplest and in a sense most natural scenarios which encodes our ignorance about physics at or near the Planck scales, the CMBR appears as a very interesting source of information which may give the first glimpses of how Nature works at its smallest - and largest - scales. We believe that the results presented are very encouraging. A careful study of present and future data should be undertaken, looking for possible modulations. It is a new kind of "exotic" feature with distinct signature that should be experimentally investigated irrespective of the state of the theoretical discussion which has not yet reached a consensus at the present time. Even a negative result will put meaningful restrictions on physics near the string or Planck scale, and provide valuable guidance to the theoretical work.

\section{Acknowledgments}

We would like to thank J. Edsjö, A. Lasenby, H. Rubinstein and U. Seljak for useful discussions. UD is a Royal Swedish Academy of Sciences Research Fellow supported by a grant from the Knut and Alice Wallenberg Foundation. The work was also supported by the Swedish Research Council (VR).

\section{References}

[1] R. H. Brandenberger, "Inflationary cosmology: Progress and problems," arXiv:hep-ph/9910410.

[2] J. Martin and R. H. Brandenberger, "The trans-Planckian problem of inflationary cosmology," Phys. Rev. D 63, 123501 (2001) arXiv:hep-th/0005209.

[3] J. C. Niemeyer, "Inflation with a high frequency cutoff," Phys. Rev. D 63, 123502 (2001) arXiv:astro-ph/0005533.

[4] R. H. Brandenberger and J. Martin, "The robustness of inflation to changes in super-Planck-scale physics," Mod. Phys. Lett. A 16, 999 (2001) arXiv:astro$\mathrm{ph} / 0005432$.

[5] A. Kempf, "Mode generating mechanism in inflation with cutoff," Phys. Rev. D 63, 083514 (2001) arXiv:astro-ph/0009209.

[6] C. S. Chu, B. R. Greene and G. Shiu, "Remarks on inflation and noncommutative geometry," Mod. Phys. Lett. A 16, 2231 (2001) arXiv:hep-th/0011241.

[7] J. Martin and R. H. Brandenberger, "A cosmological window on trans-Planckian physics," arXiv:astro-ph/0012031. 
[8] L. Mersini, M. Bastero-Gil and P. Kanti, "Relic dark energy from trans-Planckian regime," Phys. Rev. D 64, 043508 (2001) arXiv:hep-ph/0101210.

[9] J. C. Niemeyer and R. Parentani, "Trans-Planckian dispersion and scaleinvariance of inflationary perturbations," Phys. Rev. D 64, 101301 (2001) arXiv:astro-ph/0101451.

[10] A. Kempf and J. C. Niemeyer, "Perturbation spectrum in inflation with cutoff," Phys. Rev. D 64, 103501 (2001) arXiv:astro-ph/0103225.

[11] A. A. Starobinsky, "Robustness of the inflationary perturbation spectrum to trans-Planckian physics," Pisma Zh. Eksp. Teor. Fiz. 73, 415 (2001) [JETP Lett. 73, 371 (2001)] arXiv:astro-ph/0104043.

[12] R. Easther, B. R. Greene, W. H. Kinney and G. Shiu, "Inflation as a probe of short distance physics," Phys. Rev. D 64, 103502 (2001) arXiv:hep-th/0104102.

[13] M. Bastero-Gil and L. Mersini, "SN1A data and CMB of Modified Curvature at Short and Large Distances," Phys. Rev. D 65 (2002) 023502 arXiv:astroph/0107256].

[14] L. Hui and W. H. Kinney, "Short distance physics and the consistency relation for scalar and tensor fluctuations in the inflationary universe," arXiv:astro$\mathrm{ph} / 0109107$.

[15] R. Easther, B. R. Greene, W. H. Kinney and G. Shiu, "Imprints of short distance physics on inflationary cosmology," arXiv:hep-th/0110226.

[16] M. Bastero-Gil, P. H. Frampton and L. Mersini, "Modified dispersion relations from closed strings in toroidal cosmology," arXiv:hep-th/0110167.

[17] R. H. Brandenberger, S. E. Joras and J. Martin, "Trans-Planckian physics and the spectrum of fluctuations in a bouncing universe," arXiv:hep-th/0112122.

[18] J. Martin and R. H. Brandenberger, "The Corley-Jacobson dispersion relation and trans-Planckian inflation," arXiv:hep-th/0201189.

[19] J. C. Niemeyer, "Cosmological consequences of short distance physics," arXiv:astro-ph/0201511.

[20] F. Lizzi, G. Mangano, G. Miele and M. Peloso, "Cosmological perturbations and short distance physics from noncommutative geometry," arXiv:hep-th/0203099.

[21] G. Shiu and I. Wasserman, "On the signature of short distance scale in the cosmic microwave background," arXiv:hep-th/0203113.

[22] R. Brandenberger and P. M. Ho, "Noncommutative spacetime, stringy spacetime uncertainty principle, and density fluctuations," arXiv:hep-th/0203119. 
[23] S. Shankaranarayanan, "Is there an imprint of Planck scale physics on inflationary cosmology?," arXiv:gr-qc/0203060.

[24] N. Kaloper, M. Kleban, A. E. Lawrence and S. Shenker, "Signatures of short distance physics in the cosmic microwave background," arXiv:hep-th/0201158.

[25] R. H. Brandenberger and J. Martin, "On signatures of short distance physics in the cosmic microwave background," arXiv:hep-th/0202142.

[26] S. F. Hassan and M. S. Sloth, "Trans-Planckian effects in inflationary cosmology and the modified uncertainty principle," arXiv:hep-th/0204110.

[27] U. H. Danielsson, "A note on inflation and transplanckian physics," Phys. Rev. D 66, 023511 (2002) arXiv:hep-th/0203198.

[28] R. Easther, B. R. Greene, W. H. Kinney and G. Shiu, "A generic estimate of trans-Planckian modifications to the primordial power spectrum in inflation," arXiv:hep-th/0204129.

[29] U. H. Danielsson, "Inflation, holography and the choice of vacuum in de Sitter space," JHEP 0207, 040 (2002) arXiv:hep-th/0205227.

[30] J. C. Niemeyer, R. Parentani and D. Campo, "Minimal modifications of the primordial power spectrum from an adiabatic short distance cutoff," arXiv:hepth/0206149.

[31] K. Goldstein and D. A. Lowe, "Initial state effects on the cosmic microwave background and trans-planckian physics," arXiv:hep-th/0208167.

[32] U. H. Danielsson, "On the consistency of de Sitter vacua," hep-th/0210058.

[33] R. H. Brandenberger, "Trans-Planckian physics and inflationary cosmology," arXiv:hep-th/0210186.

[34] N. A. Chernikov and E. A. Tagirov, "Quantum theory of scalar field in de Sitter space-time," Ann. Inst. Henri Poincaré, vol. IX, nr 2, (1968) 109.

[35] E. Mottola, "Particle Creation In De Sitter Space," Phys. Rev. D 31 (1985) 754.

[36] B. Allen, "Vacuum States In De Sitter Space," Phys. Rev. D 32 (1985) 3136.

[37] R. Floreanini, C. T. Hill and R. Jackiw, "Functional Representation For The Isometries Of De Sitter Space," Annals Phys. 175 (1987) 345.

[38] R. Bousso, A. Maloney and A. Strominger, "Conformal vacua and entropy in de Sitter space," arXiv:hep-th/0112218.

[39] M. Spradlin and A. Volovich, "Vacuum states and the S-matrix in dS/CFT," arXiv:hep-th/0112223. 
[40] T. Banks and L. Mannelli, "De Sitter vacua, renormalization and locality," arXiv:hep-th/0209113.

[41] M. B. Einhorn and F. Larsen, "Interacting Quantum Field Theory in de Sitter Vacua," arXiv:hep-th/0209159.

[42] N. Kaloper, M. Kleban, A. Lawrence, S. Shenker and L. Susskind, "Initial conditions for inflation," arXiv:hep-th/0209231.

[43] A. A. Starobinsky and I. I. Tkachev, "Trans-Planckian particle creation in cosmology and ultra-high energy cosmic rays," arXiv:astro-ph/0207572.

[44] D. Polarski and A. A. Starobinsky, "Semiclassicality and decoherence of cosmological perturbations," Class. Quant. Grav. 13, 377 (1996) arXiv:gr-qc/9504030.

[45] A. R. Liddle and D. H. Lyth, "Cosmological inflation and large-scale structure", Cambridge University Press 2000.

[46] P. Horava and E. Witten, "Heterotic and type I string dynamics from eleven dimensions," Nucl. Phys. B 460 (1996) 506 arXiv:hep-th/9510209].

[47] P. Horava and E. Witten, "Eleven-Dimensional Supergravity on a Manifold with Boundary," Nucl. Phys. B 475 (1996) 94 [arXiv:hep-th/9603142].

[48] J. Polchinski, "String Theory. Vol. 2: Superstring Theory And Beyond," Cambridge, UK: Univ. Pr. (1998) 531 p.

[49] A. Kosowsky, "The cosmic microwave background," arXiv:astro-ph/0102402.

[50] C. B. Netterfield et al. [Boomerang Collaboration], "A measurement by BOOMERANG of multiple peaks in the angular power spectrum of the cosmic microwave background," Astrophys. J. 571, 604 (2002) arXiv:astro$\mathrm{ph} / 0104460$.

[51] S. Hanany et al., "MAXIMA-1: A Measurement of the Cosmic Microwave Background Anisotropy on angular scales of 10 arcminutes to 5 degrees," Astrophys. J. 545, L5 (2000) arXiv:astro-ph/0005123.

[52] C. Pryke, N. W. Halverson, E. M. Leitch, J. Kovac, J. E. Carlstrom, W. L. Holzapfel and M. Dragovan, "Cosmological Parameter Extraction from the First Season of Observations with DASI," Astrophys. J. 568, 46 (2002) arXiv:astro-ph/0104490.

[53] A. Benoit [the Archeops Collaboration], "The Cosmic Microwave Background Anisotropy Power Spectrum measured by Archeops," arXiv:astro-ph/0210305.

[54] E. M. Leitch et al., "Measuring Polarization with DASI," arXiv:astro$\mathrm{ph} / 0209476$. 
[55] The NASA MAP mission, homepage http://map.gsfc.nasa.gov

[56] The ESA Planck mission, homepage http://astro.estec.esa.nl/SAgeneral/Projects/Planck/

[57] L. Knox, "Determination of inflationary observables by cosmic microwave background anisotropy experiments," Phys. Rev. D 52, 4307 (1995) arXiv:astro$\mathrm{ph} / 9504054$.

[58] U. Seljak and M. Zaldarriaga, "A Line of Sight Approach to Cosmic Microwave Background Anisotropies," Astrophys. J. 469, 437 (1996) arXiv:astro$\mathrm{ph} / 9603033$.

[59] W. Hu and N. Sugiyama, "Anisotropies in the cosmic microwave background: An Analytic approach," Astrophys. J. 444, 489 (1995).

[60] J. R. Bond, G. Efstathiou and M. Tegmark, "Forecasting Cosmic Parameter Errors from Microwave Background Anisotropy Experiments," Mon. Not. Roy. Ast. Soc. 291, L33 (1997) arXiv:astro-ph/9702100.

[61] G. Jungman, M. Kamionkowski, A. Kosowsky and D. N. Spergel, "Cosmological parameter determination with microwave background maps," Phys. Rev. D 54, 1332 (1996) arXiv:astro-ph/9512139.

[62] C. H. Lineweaver, "The Cosmic Microwave Background and Observational Convergence in the $\Omega_{m}-\Omega_{\lambda}$ Plane," Astrophys. J. 505, L69 (1998) arXiv:astro$\mathrm{ph} / 9805326]$.

[63] E. J. Copeland, I. J. Grivell and A. R. Liddle, "Cosmological parameter estimation and the spectral index from inflation," Mon. Not. Roy. Ast. Soc. 298, 1233 (1998) arXiv:astro-ph/9712028.

[64] T. Souradeep, J. R. Bond, L. Knox, G. Efstathiou and M. S. Turner, "Prospects for measuring inflation parameters with the CMB," arXiv:astro-ph/9802262.

[65] S. Hannestad, "Reconstructing the inflationary power spectrum from CMBR data," Phys. Rev. D 63, 043009 (2001) arXiv:astro-ph/0009296]. 